\title{
OPTIMAL COMBINATION OF DATA MODES IN INVERSE PROBLEMS: MAXIMUM COMPATIBILITY ESTIMATE
}

\author{
Mikko Kaasalainen \\ Department of Mathematics \\ Tampere University of Technology \\ P.O. Box 553, 33101 Tampere \\ Finland
}

\begin{abstract}
We present an optimal strategy for the relative weighting of different data modes in inverse problems, and derive the maximum compatibility estimate (MCE) that corresponds to the maximum likelihood or maximum a posteriori estimates in the case of a single data mode. MCE is not explicitly dependent on the noise levels, scale factors or numbers of data points of the complementary data modes, and can be determined without the mode weight parameters. As a case study, we consider the problem of reconstructing the shape of a body in $\mathbb{R}^{3}$ from the boundary curves (profiles) and volumes (brightness values) of its generalized projections.
\end{abstract}

Keywords: Inverse problems, computational geometry, three-dimensional polytopes

\section{Introduction}

In many inverse problems, various complementary data modes are available. For example, constructing the shape model of a body in $\mathbb{R}^{3}$ is typically based on projectionlike data at various viewing geometries. In this paper, we consider the case where images $\mathcal{I}\left(\omega, \omega_{0}\right)$ (generalized projections) obtained at viewing and illumination directions $\omega, \omega_{0} \in S^{2}$ are available, but the reliable infomation in these images is only contained in the boundary curves $\partial \mathcal{I}$ between the dark background or a shadow and the illuminated portion of the target surface. This is a typical case in adaptive optics data in astrophysics, where the coverage of viewing geometries is also seldom wide enough to enable a full reconstruction of the model from images alone. ${ }^{2}$ Thus we include the possibility of augmenting the image dataset with a set of measured brightnesses (volumes of the generalized projections) $L\left(\omega, \omega_{0}\right)$ of the target at various observing geometries. 


\section{Case study: generalized projections}

We consider the inverse problem of determining the shape of a body $\mathcal{B} \in \mathbb{R}^{3}$ from some measured profiles of generalized projections $\partial \mathcal{I}\left(\omega_{i}, \omega_{0 i}\right), i=$ $1, \ldots, n$ and their volumes $L\left(\omega_{0 i}, \omega_{i}\right), i=1, \ldots, m .^{3,4}$

Our goal is to construct a total goodness-of-fit measure $\chi_{\text {tot }}^{2}$

$$
\chi_{\text {tot }}^{2}=\chi_{L}^{2}+\lambda_{\partial} \chi_{\partial}^{2}+\lambda_{R} g(P),
$$

where $L$ denotes brightness data, $\partial$ generalized profiles, and $R$ regularizing functions $g(P)$ (see Ref. 4 for discussion of these), where $P \in \mathbb{R}^{p}$ is the vector of model parameters. Determining an optimal value for $\lambda_{\partial}$ (and $\lambda_{R}$ ) is part of the inverse problem.

The volumes of generalized projections are also called total or diskintegrated brightnesses: ${ }^{3}$

$$
L\left(\omega_{0}, \omega\right)=\int_{\mathcal{A}_{+}} R\left(x ; \omega_{0}, \omega\right)\langle\omega, \nu(x)\rangle d \sigma(x),
$$

where $\mathcal{A}_{+}$is the set of visible and illuminated points $x \in \mathcal{B},{ }^{3} \nu(x) \in S^{2}$ and $d \sigma(x)$ are, respectively, the outward surface normal and surface patch of $\mathcal{B}$, and $R\left(x ; \omega_{0}, \omega\right) \in \mathbb{R}$ describes the intensity of scattered light at the point $x$ on the surface. In its basic form,

$$
\chi_{L}^{2}=\sum_{i}\left[L^{(\mathrm{obs})}\left(\omega_{0 i}, \omega_{i}\right)-L^{(\mathrm{mod})}\left(\omega_{0 i}, \omega_{i}\right)\right]^{2}
$$

(assuming a constant noise level; see Ref. 3 and references therein for modifications and variations of this). $L$-data on $S^{2} \times S^{2}$ uniquely determine a convex body and the solution is stable ${ }^{3}$ but $L$-data do not carry information on nonconvexities in most realistically available $S^{2} \times S^{2}$ geometries in practice.

For many typical adaptive optics targets in our solar system, the generalized profiles are starlike due to the proximity of $\omega$ and $\omega_{0}$ and some regularity of the target shape at global scale. ${ }^{2}$ Then we can write $\chi_{\partial}^{2}$ by considering, for each profile $i$, their observed and modelled maximal radii (from some point $\varkappa_{0} \in \mathbb{R}^{2}$ within the profile) on the projection plane at direction angles $\alpha_{i j}$ (starting from a chosen coordinate direction):

$$
\chi_{\partial}^{2}=\sum_{i j}\left[r_{\max }^{(\mathrm{obs})}\left(\alpha_{i j}\right)-r_{\max }^{(\bmod )}\left(\alpha_{i j}\right)\right]^{2} .
$$

We now represent the body $\mathcal{B}$ as a polytope. Let two vertices $a$ and $b$ of a facet have projection points $\varkappa_{a}, \varkappa_{b}$. The intersection point $\varkappa$ of the radius line at $\alpha$ and the projection of the facet edge $a b$ is readily determined. The 
model $r_{\max }(\alpha)$ can now be determined by going through all eligible facet edges and their intersection points $\varkappa_{a b}(\alpha)$ :

$$
r_{\max }^{(\bmod )}(\alpha)=\max \left\{\left\|\varkappa_{a b}(\alpha)-\varkappa_{0}\right\| \mid a, b \in \mathcal{V}_{+}\right\},
$$

where $\mathcal{V}_{+}$is the set of vertices of the set of facets $\tilde{\mathcal{A}}_{+}$approximating $\mathcal{A}_{+}$. The set $\tilde{\mathcal{A}}_{+}$is determined by ray-tracing. ${ }^{3}$ In general, facet edge circuits $\partial \tilde{\mathcal{A}}_{+}$ approximating $\partial \mathcal{A}_{+}$(and corresponding forms of $\chi_{\partial}^{2}$ ) can be automatically derived for non-starlike shape models or profiles as well. ${ }^{4}$

\section{Maximum compatibility estimate}

Let us choose as goodness-of-fit measures (from which probability distributions can be constructed) the $\chi^{2}$-functions of $n$ data modes. Our task is to construct a joint $\chi_{\text {tot }}$ with well-defined weighting for each data mode:

$$
\chi_{\text {tot }}^{2}(P, D)=\chi_{1}^{2}\left(P, D_{1}\right)+\sum_{i=2}^{n} \lambda_{i-1} \chi_{i}^{2}\left(P, D_{i}\right) \quad D=\left\{D_{i}, i=1, \ldots, n\right\}
$$

(to which regularization functions $g(P)$ can be added), where $D_{i}$ denotes the data from the source $i$, and $P \in \mathbb{R}^{p}$ is the set of model parameter values. We assume the $\chi_{i}^{2}$-space to be nondegenerate, i.e.,

$$
\arg \min \chi_{i}^{2}(P) \neq \arg \min \chi_{j}^{2}(P), \quad i \neq j .
$$

In two dimensions, denote

$$
\begin{aligned}
& x(\lambda):=\left\{\chi_{1}^{2} \mid \min \chi_{\mathrm{tot}}^{2} ; \lambda\right\}, \\
& y(\lambda):=\left\{\chi_{2}^{2} \mid \min \chi_{\mathrm{tot}}^{2} ; \lambda\right\} .
\end{aligned}
$$

The curve

$$
\mathcal{S}(\lambda):=[\log x(\lambda), \log y(\lambda)]
$$

resembles the well-known "L-curve" related to, e.g., Tikhonov regularization. ${ }^{1,6}$ However, here we make no assumptions on the shape of $\mathcal{S}$. The curve $\mathcal{S}$ is a part of the boundary $\partial \mathcal{R}$ of the region $\mathcal{R} \in \mathbb{R}^{2}$ formed by the mapping $\chi: \mathbb{R}^{p} \rightarrow \mathbb{R}^{2}$ from the parameter space $\mathbb{P}$ into $\chi_{i}^{2}$-space:

$$
\chi=\left\{\mathbb{P} \rightarrow\left(\log \chi_{1}^{2}, \log \chi_{2}^{2}\right)\right\}, \quad \mathcal{R}=\chi(\mathcal{P})
$$

where the set $\mathcal{P}$ includes all the possible values of model parameters (assuming that $\chi$ is continuous and well-behaved such that a connected $\mathcal{R}$ and $\partial \mathcal{R}$ exist). If the possible values of $\chi_{i}^{2}$ are not bounded, the remaining part $\partial \mathcal{R} \backslash \mathcal{S}$ stretches droplet-like towards $(\infty, \infty)$. The parameter $\lambda$ describes 
the position on the interesting part $\mathcal{S} \subset \partial \mathcal{R}$, and it is up to us to define a criterion for choosing the optimal value of $\lambda$.

The logarithm ensures that the shape of $\mathcal{S}(\lambda)$ is invariant under unit or scale transforms in the $\chi_{i}^{2}$ as they merely translate $\mathcal{S}$ in the $\left(\log \chi_{1}^{2}, \log \chi_{2}^{2}\right)$ plane. It also provides a meaningful metric for the $\log \chi_{i}^{2}$-space: distances depict the relative difference in $\chi^{2}$-sense, removing the problem of comparing the absolute values of quite different types of $\chi_{i}^{2}$. The endpoints of $\mathcal{S}(\lambda)$ are at $\lambda=0$ and $\lambda=\infty$, i.e., at the values of $\chi_{i}^{2}$ that result from using only one of the data modes in inversion. We can translate the origin of the $\left(\log \chi_{1}^{2}, \log \chi_{2}^{2}\right)$-plane to a more natural position by choosing the new coordinate axes to pass through these endpoints. Denote

$$
\begin{aligned}
& \hat{x}_{0}=\left.\log x(\lambda)\right|_{\lambda=0}=\log \min \chi_{1}^{2} \\
& \hat{y}_{0}=\left.\log y(\lambda)\right|_{\lambda \rightarrow \infty}=\log \min \chi_{2}^{2} .
\end{aligned}
$$

Then the "ideal point" $\left(\hat{x}_{0}, \hat{y}_{0}\right)$ is the new origin in the $(\log x, \log y)$-plane. A natural choice for an optimal location on $\mathcal{S}$ is the point closest to $\left(\hat{x}_{0}, \hat{y}_{0}\right)$, i.e., the parameter values $P_{0} \in \mathbb{P}$ such that

$$
P_{0}=\arg \min \left(\left[\log \chi_{1}^{2}(P)-\hat{x}_{0}\right]^{2}+\left[\log \chi_{2}^{2}(P)-\hat{y}_{0}\right]^{2}\right),
$$

so we have, with $\lambda$ as argument,

$$
\lambda_{0}=\arg \min \left(\left[\log x(\lambda)-\hat{x}_{0}\right]^{2}+\left[\log y(\lambda)-\hat{y}_{0}\right]^{2}\right) .
$$

In this approach, neither the numbers of data points in each $\chi_{i}^{2}$ nor the noise levels as such affect the solution for the optimal $P_{0}$ as their scaling effects cancel out in each quadratic term. $P_{0}$ is thus a pure compatibility estimate describing the best model compromise explaining the datasets of different modes simultaneously.

We call the point $P_{0}$ the maximum compatibility estimate (MCE), and $\lambda_{0}$ the maximum compatibility weight (MCW). This corresponds to the maximum likelihood estimate in the case of one data mode, or to the maximum a posteriori estimate as well since we can include regularization functions here. If regularizing is used, the weights for the functions are either determined in a similar manner (see below), or they can be fixed and the regularization terms are absorbed in $\chi_{1}^{2}$ (otherwise $\mathcal{S} \subset \partial \mathcal{R}$ does not hold).

Another choice, frequently used in the L-curve approach, is to find the $\lambda$ at which $\mathcal{S}$ attains its maximum curvature, ${ }^{1,6}$ but evaluating this point is less robust than finding $\lambda_{0}$, and (11) is a more natural prescription, requiring no assumptions on the shape of $\mathcal{S}$. We make two implicit assumptions here: 
(1) The solutions $P_{\partial \mathcal{R}}$ corresponding to points on $\partial \mathcal{R}$ should be continuous (and one-to-one) in $\mathbb{P}$-space along $\partial \mathcal{R}$ at least in the vicinity of the solution corresponding to $\lambda_{0}$. If this is not true (in practice, if $P_{\lambda}=$ $\arg \min \chi_{\text {tot }}^{2}(P)$ makes large jumps in $\mathbb{P}$ for various $\lambda$ around $\left.\lambda_{0}\right)$, one should be cautious about the uniqueness and stability of the chosen solution $P_{0}$, and restrict the regions of $\mathbb{P}$ included in the analysis.

(2) The optimal point $\lambda_{0}$ on $\mathcal{S}$ should be feasible: if we have upper limits $\epsilon_{i}$ to acceptable $\chi_{i}^{2}$, the feasible region $\mathcal{F}$ is the rectangle $\bigcap_{i}\left\{\log \chi_{i}^{2} \leq\right.$ $\left.\log \epsilon_{i}\right\}$. If $\left[\log \chi_{1}^{2}\left(P_{0}\right), \log \chi_{2}^{2}\left(P_{0}\right)\right] \notin \mathcal{F}$ and $\mathcal{F} \cap \mathcal{R} \neq \emptyset$, we choose the point on the portion $\mathcal{S} \subset \mathcal{R}$ closest to the one corresponding to $\lambda_{0}$ (i.e., $\log \chi_{i}^{2}=\log \epsilon_{i}$ for one $i$ ). If $\mathcal{F} \cap \mathcal{R}=\emptyset$, the data modes do not allow a compatible joint model, so either the model is incorrect for one or both data modes, or one or both $\epsilon_{i}$ have been estimated too low (e.g., systematic errors have not been taken into account). Note that model insufficiency should be taken into account in the estimation of $\epsilon_{i}$.

Note that, in the interpretation $\mathcal{R}=\chi(\mathcal{P}), \lambda, \chi_{\text {tot }}^{2}$ and $\partial \mathcal{R}$ are all in fact superfluous quantities, and we can locate the point estimate MCE $P_{0}$ entirely without them with standard optimization procedures (and with no extra computational cost). However, it is useful (though computationally somewhat noisier) to approximate $\mathcal{S}$ via the minimization of $\chi_{\text {tot }}^{2}$ with sample values of $\lambda$ (see Fig. 1a), as in addition to obtaining the MCW $\lambda_{0}$ (and hence MCE as well) we can plot $\mathcal{S}$ to examine the mutual behaviour of the complementary data sources (including the position of the feasibility region $\mathcal{F}$ w.r.t. $\mathcal{S}$ ). The solution for $\lambda_{0}$ is also needed for constructing distributions based on $\chi_{\text {tot }}^{2}$. Another possibility to examine $\mathcal{R}$ and $\partial \mathcal{R}$ is direct adaptive Monte Carlo sampling, but this is computationally slow.

This approach straightforwardly generalizes to $n \chi^{2}$-functions and $n-1$ parameters $\lambda_{i}$ describing the position on the $n$-1-dimensional boundary surface $\partial \mathcal{R}$ of an $n$-dimensional domain $\mathcal{R}$ : the MCE is

$$
P_{0}=\arg \min \sum_{i=1}^{n}\left[\log \frac{\chi_{i}^{2}(P)}{\chi_{i 0}^{2}}\right]^{2}, \quad \chi_{i 0}^{2}:=\min \chi_{i}^{2}(P),
$$

and the MCW is, for $\lambda \in \mathbb{R}^{n-1}$,

$$
\lambda_{0}=\arg \min \sum_{i=1}^{n}\left[\log \frac{\hat{\chi}_{i, \mathrm{tot}}^{2}(\lambda)}{\chi_{i 0}^{2}}\right]^{2}, \quad \hat{\chi}_{i, \text { tot }}^{2}(\lambda):=\left\{\chi_{i}^{2} \mid \min \chi_{\text {tot }}^{2} ; \lambda\right\} .
$$

Another scale-invariant version of MCE can be constructed by plotting 
$\chi_{i}^{2}$ in units of $\chi_{i}^{2} / \chi_{i 0}^{2}$ and shifting the new origin to $\chi_{i}^{2} / \chi_{i 0}^{2}=1$ :

$$
P_{0}=\arg \min \sum_{i=1}^{n}\left[\frac{\chi_{i}^{2}(P)}{\chi_{i 0}^{2}}-1\right]^{2}, \quad \lambda_{0}=\arg \min \sum_{i=1}^{n}\left[\frac{\hat{\chi}_{i, \text { tot }}^{2}(\lambda)}{\chi_{i 0}^{2}}-1\right]^{2} .
$$

This, however, is exactly the first-order approximation of (12) and (13) in $\delta \ll 1$ when $\chi_{i}^{2} / \chi_{i 0}^{2}=1+\delta$, giving virtually the same result as (12) and (13) as usually $\chi_{i}^{2}\left(P_{0}\right) / \chi_{i 0}^{2}-1 \ll 1$ in the region around $\chi_{i}^{2}\left(P_{0}\right)$, and any larger ratios of $\chi_{i}^{2} / \chi_{i 0}^{2}$ are not eligible for the optimal solution (see Fig. 1a).

It is possible to use this approach for general regularizing functions $g(P)$ as well (change $\chi_{i}^{2} \rightarrow g(P)$ for some $i$ ), but in such cases the shape of $\mathcal{S}$ must be taken into account. If it is possible to have a solution $g\left(P^{\prime}\right)=0$ for a regularizing function $g$ (or an almost vanishing $g\left(P^{\prime}\right)$ such that $\log g\left(P^{\prime}\right) \rightarrow$ $-\infty)$, one should, e.g., set a lower practical limit to $g(P)$ by looking at the shape of $S$, and choose the $\lambda_{0}$ within the restricted part of $S$.

\section{Numerical implementation}

As examples of the optimal combining of brightness values and profile contours, we show some results for asteroid data.

Fig. 1a depicts a typical evaluation of the curve $\mathcal{S}$ for 2 Pallas at various choices of $\lambda$ (or rather, this plot portrays the cross-section of the 2 -surface $\partial \mathcal{R}$ in $\mathbb{R}^{3}$ with smoothness regularization weight fixed at its final optimal value). The values for $\chi_{i}^{2}$ are normalized to be the rms deviations of model fits $d_{i}=\sqrt{\chi_{i}^{2} / N_{i}}$, as in logarithmic scale this corresponds only to a shift of origin and a uniform linear change of plot scaling. The plotted points outline the curve $\mathcal{S}(\lambda)$ that is rather an oblique line than an L-shape, and the ideal point region, i.e., the point closest to the lower left-hand corner, can directly be found. The endpoints $\lambda=0$ and $\lambda=\infty$ stop at saturation regions rather than continue to large distances in the $\log \chi^{2}$-space. As can be seen from Fig. 1a, computational noise in the estimated points at $\lambda=0$ and $\lambda=\infty$, corresponding to a small change of the position of the new origin w.r.t. $\mathcal{S}$, does not affect the estimated location of the optimal point on $\mathcal{S}$ significantly.

A sample observed vs. modelled profiles for 41 Daphne is shown in Fig. 1b. The starlike surface model was described by the exponential Laplace (spherical harmonics) series for the surface radius $r$ (for explicit positivity):

$$
r(\theta, \varphi)=\exp \left[\sum_{l m} c_{l m} Y_{l}^{m}(\theta, \varphi)\right], \quad(\theta, \varphi) \in S^{2},
$$



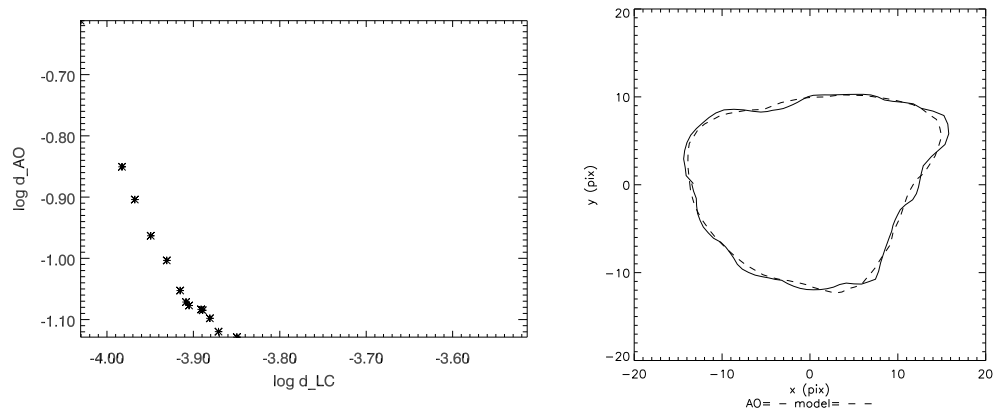

Fig. 1. (a) $\mathcal{S}$ curve plotted for 2 Pallas with various weights $\lambda$ (LC for brightness data, AO for adaptive optics profiles). (b) Sample observed (solid line) vs. modelled (dashed line) $\mathrm{AO}$ profile contour for 41 Daphne. Coordinates are in pixel units.

truncated at $l=8, m=6$, with $c_{l m}$ as the shape parameters to be solved for. Other model parameters are the profile offset $\varkappa_{0}$ for each image and the physical spin parameters describing the rotational transformations yielding the correct viewing and illumination directions $\left(\omega, \omega_{0}\right)$ on the body. ${ }^{3-5}$

\section{Discussion}

The concept of the maximum compatibility estimate is directly applicable to any inverse problems with complementary data modes. The invariance properties of the MCE make it more generally usable than heuristic strategies for choosing the weights, especially when they use assumptions on the shape of $\partial \mathcal{R}$ or other case-specific characteristics. In our case study, the use of profiles is practical as it removes two sources of systematic errors inherent to using full images (brightness distributions $\mathcal{I}$ on the image plane): the errors in $\mathcal{I}$ from adaptive optics deconvolution and the model $\mathcal{I}$ errors due to the insufficently modellable light-scattering properties of the surface of the target body.

\section{References}

1. M. Belge, M. Kilmer, and E. Miller, Inverse Problems 18, 1161 (2002).

2. B. Carry, C. Dumas, M. Kaasalainen, and 9 colleagues, Icarus, in press (2009).

3. M. Kaasalainen and L. Lamberg, Inverse Problems 22, 749 (2006).

4. M. Kaasalainen, Inverse Problems and Imaging, submitted (2009).

5. M. Kaasalainen, J. Durech, B. Warner, Y. Krugly, and N. Gaftonyuk, Nature 446, 420 (2007).

6. J. Kaipio and E. Somersalo, Statistical and computational inverse problems (Springer, New York 2005). 